\title{
Nonclassical Contact Symmetries and Charpit's Method of Compatibility
}

\author{
Daniel J ARRIGO
}

Department of Mathematics, University of Central Arkansas, Conway AR 72035 USA

E-mail: darrigo@uca.edu

Received June 22, 2004; Accepted December 2, 2004

\begin{abstract}
Charpit's method of compatibility and the method of nonclassical contact symmetries for first order partial differential equation are considered. It is shown that these two methods are equivalent as Charpit's method leads to the determining equations arising from the method of nonclassical contact symmetries. Several examples are considered illustrating how Charpit's method leads quickly and easily to nonclassical contact symmetries.
\end{abstract}

\section{Introduction}

The study of nonlinear partial differential equations (PDEs) is one of the most important problems in applied mathematics and mathematical physics. Since most real world problems are modeled by equations of this type, a search for their solutions continues to be a topic of research. As is often the case, interesting and/or unusual behavior has been attributed to the nonlinearities within the model equations. For example, "waiting-time," finite time "blow-up," and extinction were all identified by simple exact solutions to the governing nonlinear partial differential equation (see [6], [5] and [12]). Even though most real world phenomena are modeled by equations that are beyond first order, first order nonlinear PDEs still continues to play an important role in modeling applications in the physical sciences. For example, the eikonal equation

$$
u_{x}^{2}+u_{y}^{2}+u_{z}^{2}=n^{2}(x, y, z)
$$

arises in geometric optics [11], in acoustics [10] and in wave propagation in reactiondiffusion systems [8].

The method typically used to construct exact solutions to first order PDEs is the method of generalized characteristics sometimes referred as Charpit's method. This method is most easily derived by compatibility. Given a first order PDE

$$
F\left(t, x, u, u_{t}, u_{x}\right)=0,
$$

a second first order PDE

$$
G\left(t, x, u, u_{t}, u_{x}\right)=0,
$$


is compatible with (1.2) if the condition

$$
F_{p} G_{t}+F_{q} G_{x}+\left(p F_{p}+q F_{q}\right) G_{u}-\left(F_{t}+p F_{u}\right) G_{p}-\left(F_{x}+q F_{u}\right) G_{q}=0,
$$

is satisfied (see, for example Kells [9]). As usual, we have used the notation $p=u_{t}$ and $q=u_{x}$.

For example, if we consider

$$
u_{t}=u_{x}^{2},
$$

then

$$
F=p-q^{2}
$$

and from (1.4), compatible equations are those that satisfy

$$
G_{t}-2 q G_{x}+\left(p-2 q^{2}\right) G_{u}=0 .
$$

Equation (1.6) is linear and the solution is easily found using the method of characteristics, namely

$$
G=G(p, q, 2 t q+x, t p+u) .
$$

As a particular example, we consider

$$
G=p+2 t q+x,
$$

giving the compatible equation

$$
u_{t}+2 t u_{x}=-x .
$$

Solving (1.9) gives

$$
u=-x t+\frac{2}{3} t^{3}+f\left(x-t^{2}\right)
$$

and substituting into (1.5) leads to the differential equation

$$
f^{\prime 2}+\lambda=0
$$

where $\lambda=x-t^{2}$ and $f=f(\lambda)$. Alternatively, we could have chosen

$$
G=t p+q+u,
$$

giving the compatible equation

$$
t u_{t}+u_{x}=-u \text {. }
$$

Solving (1.13) gives

$$
u=\frac{f(x-\ln |t|)}{t},
$$


and substituting into (1.5) leads to the differential equation

$$
f^{\prime 2}+f^{\prime}+f=0
$$

where $\lambda=x-\ln |t|$ and $f=f(\lambda)$. What is important to note is that the solution of a compatible equations, (1.9) and (1.13), reduces the original equation, (1.5), to the ordinary differential equations, (1.11) and (1.15), respectively. The fact that this reduction occurs is not a coincidence! It is well known that symmetry methods lead to reductions of partial differential equations (see Bluman and Kumei [3]). In this paper, we will show that Charpit's method for constructing compatible equations to first order partial differential equations is equivalent to calculating their nonclassical contact symmetries, thus explaining why the reduction process occurs using Charpit's method.

The paper is organized as follows. In section 2, nonclassical contact symmetries are constructed for arbitrary first order PDEs. Using their connection to nonclassical LieBacklünd symmetries, Charpit's equation is derived demonstrating their equivalence. In section 3, several examples will be considered illustrating this method of compatibility.

Finally we note that for simplicity, our focus is on first order partial differential equations in only two independent variables. However, the results presented in this paper are easily extended to first order equations in $n$ independent variables.

\section{Contact symmetries}

Equation (1.2) is invariant under the one-parameter Lie group of contact transformations

$$
\begin{aligned}
& \bar{t}=t+T\left(t, x, u, u_{t}, u_{x}\right) \varepsilon+O\left(\varepsilon^{2}\right), \\
& \bar{x}=x+X\left(t, x, u, u_{t}, u_{x}\right) \varepsilon+O\left(\varepsilon^{2}\right), \\
& \bar{u}=u+U\left(t, x, u, u_{t}, u_{x}\right) \varepsilon+O\left(\varepsilon^{2}\right),
\end{aligned}
$$

if

$$
\left.\Gamma(F)\right|_{F=0}=0,
$$

where the infinitesimal generator $\Gamma$ is given by

$$
\Gamma=T \frac{\partial}{\partial t}+X \frac{\partial}{\partial x}+U \frac{\partial}{\partial u}+U_{[t]} \frac{\partial}{\partial u_{t}}+U_{[x]} \frac{\partial}{\partial u_{x}} .
$$

In (2.3), the functions $T, X$ and $U$ are subject to the contact condition

$$
U_{p}-T_{p} u_{t}-X_{p} u_{x}=0, \quad U_{q}-T_{q} u_{t}-X_{q} u_{x}=0,
$$

and $U_{[t]}$ and $U_{[x]}$ are given by

$$
\begin{gathered}
U_{[t]}=D_{t} U-u_{t} D_{t} T-u_{x} D_{t} X, \\
U_{[x]}=D_{x} U-u_{t} D_{x} T-u_{x} D_{x} X,
\end{gathered}
$$


where the total differential operators $D_{t}$ and $D_{x}$ are given, respectively, by

$$
\begin{aligned}
D_{t} & =\frac{\partial}{\partial t}+u_{t} \frac{\partial}{\partial u}+u_{t t} \frac{\partial}{\partial u_{t}}+u_{t x} \frac{\partial}{\partial u_{x}}+\cdots, \\
D_{x} & =\frac{\partial}{\partial x}+u_{x} \frac{\partial}{\partial u}+u_{t x} \frac{\partial}{\partial u_{t}}+u_{x x} \frac{\partial}{\partial u_{x}}+\cdots
\end{aligned}
$$

We now state the main result of the paper.

Theorem 1. The determining equations for the nonclassical symmetries of the first order $P D E$

$$
F\left(t, x, u, u_{t}, u_{x}\right)=0,
$$

under the contact transformation group with the infinitesimal generator

$$
\Gamma=T \frac{\partial}{\partial t}+X \frac{\partial}{\partial x}+U \frac{\partial}{\partial u}+U_{[t]} \frac{\partial}{\partial u_{t}}+U_{[x]} \frac{\partial}{\partial u_{x}},
$$

is equivalent to Charpit's method.

Proof. Consider the Lie-Backlünd transformation with the infinitesimal generator

$$
G\left(t, x, u, u_{t}, u_{x}\right) \frac{\partial}{\partial u} .
$$

This is equivalent to a contact transformation group with the infinitesimal generator

$$
\Gamma=T \frac{\partial}{\partial t}+X \frac{\partial}{\partial x}+U \frac{\partial}{\partial u}+U_{[t]} \frac{\partial}{\partial u_{t}}+U_{[x]} \frac{\partial}{\partial u_{x}},
$$

provided

$$
\begin{array}{r}
T=\frac{\partial G}{\partial u_{t}}, \quad X=\frac{\partial G}{\partial u_{x}}, \\
U=u_{t} \frac{\partial G}{\partial u_{t}}+u_{x} \frac{\partial G}{\partial u_{x}}-G, \\
U_{[t]}=-\frac{\partial G}{\partial t}-u_{t} \frac{\partial G}{\partial u}, \quad U_{[x]}=-\frac{\partial G}{\partial x}-u_{x} \frac{\partial G}{\partial u},
\end{array}
$$

(see Bluman and Kumei [3]). Invariance of any first order PDE

$$
F\left(t, x, u, u_{t}, u_{x}\right)=0
$$

is given by

$$
T \frac{\partial F}{\partial t}+X \frac{\partial F}{\partial x}+U \frac{\partial F}{\partial u}+U_{[t]} \frac{\partial F}{\partial u_{t}}+U_{[x]} \frac{\partial F}{\partial u_{x}}=0,
$$

subject to $F=0$. Substituting (2.11) into (2.13) and expanding gives

$$
\begin{aligned}
G_{p} F_{t} & +G_{q} F_{x}+p G_{p} F_{u}+q G_{q} F_{u}-G F_{u} \\
& -G_{t} F_{p}-p G_{u} F_{p}-G_{x} F_{q}-q G_{u} F_{q}=0,
\end{aligned}
$$


noting again the use of the notation $p=u_{t}$ and $q=u_{x}$. Classical contact symmetries requires $\left.\Gamma(F)\right|_{F=0}=0$. Nonclassical contact symmetries requires further $\left.\Gamma(F)\right|_{F=0, G=0}=$ 0 , so that $G F_{u}=0$ in (2.14). Re-arranging (2.14) gives

$$
F_{p} G_{t}+F_{q} G_{x}+\left(p F_{p}+q F_{q}\right) G_{u}-\left(F_{t}+p F_{u}\right) G_{p}-\left(F_{x}+q F_{u}\right) G_{q}=0,
$$

which is Charpit's equation (1.4). Conversely, if we begin with Charpit's equation, (2.15) and re-organize, then we can obtain (2.14) if we impose $G F_{u}=0$. This is identical to the invariance condition of $F\left(t, x, u, u_{t}, u_{x}\right)=0$ under the contact transformation group generated by the infinitesimal operator as given in (2.10).

\section{$3 \quad$ Examples}

In this section we consider two examples illustrating how Charpit's method gives rise to a reduction of the original equation.

Example 1, $u_{t}=u_{x}^{2}$

As seen in the introduction of the paper, the PDE

$$
u_{t}=u_{x}^{2}
$$

admits compatible equations

$$
G(p, q, 2 t q+x, t p+u)=0 .
$$

As particular examples we considered equations (1.9) and (1.13). It is interesting to note that both of these equations also arise from classical Lie group analysis. The Lie group of infinitesimal transformations leaving (3.1) invariant is generated by

$$
\Gamma=T \frac{\partial}{\partial t}+X \frac{\partial}{\partial x}+U \frac{\partial}{\partial u}
$$

where

$$
\begin{aligned}
T & =-4 c_{1} t^{2}+c_{4} x t+c_{5} t+c_{8} x^{2}+c_{9} x+c_{10}, \\
X & =-4 c_{1} x t-2 c_{2} t+c_{4}\left(\frac{1}{2} x^{2}-2 t u\right)+c_{6} x+c_{7}-4 c_{8} x u-2 c_{9} u, \\
U & =c_{1} x^{2}+c_{2} x+c_{3}+c_{4} x u-c_{5} u+2 c_{6} u-4 c_{8} u^{2},
\end{aligned}
$$

where $c_{1}-c_{10}$ are arbitrary constants (see, for example, Hydon [7]). The invariant surface condition associated with (3.1) is

$$
T u_{t}+X u_{x}=U .
$$

We note that choosing $c_{2}=-1$ and $c_{10}=1$ and the remaining constants to zero in (3.4) shows that (3.5) recovers (1.9). Choosing $c_{5}=1$ and $c_{7}=1$ and the remaining constants to zero in (3.4) shows that (3.5) recovers (1.13). In fact, the invariant surface condition (3.5) with $T, X$ and $U$ as given in (3.4) can be recovered from (3.2) by considering

$$
\begin{aligned}
G(p, q, r, s) & +2\left(p-q^{2}\right)\left(c_{4} r-4 c_{8} s+c_{6}\right) t \\
& +2\left(p-q^{2}\right)\left(2 c_{8} p-c_{4} q-2 c_{1}\right) t^{2}=0,
\end{aligned}
$$


and choosing $G$ as

$$
\begin{aligned}
G(p, q, r, s) & =-c_{1} r^{2}-c_{2} r-c_{3}+c_{4}\left(\frac{1}{2} q r^{2}-r s\right)+c_{5} s+c_{6}(q r-2 s) \\
& +c_{7} q+c_{8}\left(p r^{2}+4 s^{2}-4 r s q\right)+c_{9}(p r-2 q s)+c_{10} p,
\end{aligned}
$$

thus illustrating that the classical Lie symmetries of (3.1) can be quickly and easily obtained from (3.2). To show that the solution of the general equation (3.2) reduces (3.1) to an ODE is to show that the solution of (3.1) reduces the general equation (3.2) to an ODE. Under the Ampére transformation

$$
t=T, \quad x=U_{X}, \quad u=X U_{X}-U,
$$

equation (3.1) becomes

$$
U_{T}=-X^{2} .
$$

Integrating with respect to $T$ gives the general solution as

$$
U=-X^{2} T+U_{0}(X)
$$

where $U_{0}(X)$ is an arbitrary function of its argument. This, in turn, gives the general solution of (3.1) parametrically as

$$
t=T, \quad x=U_{0}^{\prime}(X)-2 X T, \quad u=X U_{0}^{\prime}(X)-U_{0}(X)-X^{2} T .
$$

Further, substitution of (3.10) into the general compatible equation (3.2) gives rise to the reduced equation

$$
G\left(U_{0}^{\prime}(X), X U_{0}^{\prime}(X)-U_{0}(X), X^{2}, X\right)=0 .
$$

Example 2, $u_{x}^{2}+u_{y}^{2}+u_{z}^{2}=a x^{2}+b y^{2}+c z^{2}+1, \quad a, b, c \in \mathbb{R}, \quad a \neq b \neq c \neq 0$

A classical symmetry analysis of equation (1.1) was considered by Borovskikh [4] of which this is a member. From his results, we find that the only classical symmetry for this example is translation in $u$. We set

$$
F=p^{2}+q^{2}+r^{2}-a^{2} x^{2}-b^{2} y^{2}-c^{2} z^{2}-1,
$$

where in this case we are using the notation $p=u_{x}, q=u_{y}$ and $r=u_{z}$. Charpit's equation is

$$
p G_{x}+q G_{y}+r G_{z}+\left(p^{2}+q^{2}+r^{2}\right) G_{u}+a^{2} x G_{p}+b^{2} y G_{q}+c^{2} z G_{r}=0,
$$

which is solved by the method of characteristics. For simplicity, we will assume $G_{u}=0$. This gives compatible equations in the form

$$
G=G\left(p^{2}-a^{2} x^{2}, q^{2}-b^{2} y^{2}, r^{2}-c^{2} z^{2},(p+a x)^{b}(q+b y)^{-a},(p+a x)^{c}(r+c z)^{-a}\right) .
$$


We now consider two particular examples.

If

$$
p^{2}-a^{2} x^{2}=k^{2}
$$

for some arbitrary constant $k$, then

$$
u_{x}=\sqrt{a^{2} x^{2}+k^{2}},
$$

(we have suppressed the negative case) which integrates to give

$$
u=\frac{1}{2} x \sqrt{a^{2} x^{2}+k^{2}}+\frac{k^{2}}{2 a} \ln \left|a x+\sqrt{a^{2} x^{2}+k^{2}}\right|+v(y, z) .
$$

Substitution into the original equation gives

$$
v_{y}^{2}+v_{z}^{2}=b^{2} y^{2}+c^{2} z^{2}-k^{2},
$$

an equation with one less independent variable! We note that this reduction could have been easily obtained if one chose separable solutions of the form

$$
u=v(y, z)+w(x),
$$

but we will show that this reduction is in fact is a nonclassical symmetry originally introduced by Bluman and Cole [2]. We associate with the invariant surface condition (3.14), the infinitesimal transformations

$$
\begin{aligned}
& \bar{x}=x+\varepsilon+O\left(\varepsilon^{2}\right), \quad \bar{y}=y+O\left(\varepsilon^{2}\right), \\
& \bar{z}=z+O\left(\varepsilon^{2}\right), \quad \bar{u}=u+\sqrt{a^{2} x^{2}+k^{2}} \varepsilon+O\left(\varepsilon^{2}\right),
\end{aligned}
$$

from which we obtain the finite transformations as

$$
\begin{aligned}
\bar{x} & =x+\varepsilon, \quad \bar{y}=y, \quad \bar{z}=z, \\
\bar{u} & =u+\frac{1}{2}(x+\varepsilon) \sqrt{a^{2}(x+\varepsilon)^{2}+k^{2}}-\frac{1}{2} x \sqrt{a^{2} x^{2}+k^{2}} \\
& +\frac{k^{2}}{2 a} \ln \left|a(x+\varepsilon)+\sqrt{a^{2}(x+\varepsilon)^{2}+k^{2}}\right|-\frac{k^{2}}{2 a} \ln \left|a x+\sqrt{a^{2} x^{2}+k^{2}}\right| .
\end{aligned}
$$

If we consider

$$
\bar{u}_{\bar{x}}^{2}+\bar{u}_{\bar{y}}^{2}+\bar{u}_{\bar{z}}^{2}=a^{2} \bar{x}^{2}+b^{2} \bar{y}^{2}+c^{2} \bar{z}^{2}+1,
$$

then under (3.18), we obtain

$u_{x}^{2}+u_{y}^{2}+u_{z}^{2}+2\left(u_{x}-\sqrt{a^{2} x^{2}+k^{2}}\right)\left(\sqrt{a^{2}(x+\varepsilon)^{2}+k^{2}}-\sqrt{a^{2} x^{2}+k^{2}}\right)=a^{2} x^{2}+b^{2} y^{2}+c^{2} z^{2}+1$,

which, by virtue of (3.14), gives the original equation thus illustrating that (3.18) is a nonclassical symmetry.

As a second example, we consider

$$
(p+a x)^{b}(q+b y)^{-a}=k,
$$


where again, $k$ is some arbitrary constant. It is convenient to introduce the new variable $v$ where

$$
v=u+\frac{1}{2}\left(a x^{2}+b y^{2}\right),
$$

thus, reducing the invariant surface condition and original equation to

$$
v_{x}=k v_{y}^{a / b}, \quad v_{x}^{2}+v_{y}^{2}+v_{z}^{2}-2 a x v_{x}-2 b y v_{y}=c^{2} z^{2}+1 .
$$

Under the Ampére transformation

$$
x=X, \quad y=V_{Y}, \quad z=Z, \quad v=Y V_{Y}-V,
$$

(3.22) becomes

$$
V_{X}=-k Y^{a / b}, \quad V_{X}^{2}+V_{Z}^{2}+2 a X V_{X}-2 b Y V_{Y}+Y^{2}=c^{2} Z^{2}+1 .
$$

Integration of the first of (3.24) leads to

$$
V=-k X Y^{a / b}+W(Y, Z),
$$

and substitution into the second of (3.24) gives the reduced equation

$$
W_{Z}^{2}-2 b Y W_{Y}+Y^{2}+k^{2} Y^{2 a / b}-c^{2} Z^{2}-1=0 .
$$

This can be further simplified under the contact transformation

$$
Y=e^{2 b W_{Y^{*}}^{*}}, \quad Z=Z^{*}, \quad W=Y^{*} W_{Y^{*}}^{*}-W^{*}+\frac{1}{4 b} e^{4 b W_{Y^{*}}^{*}}+\frac{k^{2}}{4 a} e^{4 a W_{Y^{*}}^{*}}
$$

giving rise to

$$
W_{Z^{*}}^{* 2}=c^{2} Z^{* 2}+Y^{*}+1,
$$

and equation that is easily solved; namely

$$
W^{*}= \pm \frac{1}{2} Z^{*} \sqrt{c^{2} Z^{* 2}+Y^{*}+1} \pm \frac{Y^{*}+1}{2 c} \ln \left|c Z^{*}+\sqrt{c^{2} Z^{* 2}+Y^{*}+1}\right|+W_{0}\left(Y^{*}\right),
$$

where $W_{0}\left(Y^{*}\right)$ is an arbitrary function of $Y^{*}$. Passing $W^{*}$ back through (3.26), (3.25), (3.23) and (3.21) gives rise to an exact solution to the original equation.

\section{Conclusion}

In this paper we have considered Charpit's method for deriving compatibility equations to a given first order PDE. We have shown that these compatible equations are equivalent to the determining equations for the nonclassical contact symmetries of the original equation. The latter is based upon the invariance of a particular equation under an infinitesimal group of contact transformations. As demonstrated through several examples, Charpit's method lead quickly and easily the determining equations. Several examples 
were considered illustrating how the original equation can be reduced to a first order PDE in one less independent variable.

This now leads to a very interesting question. Can the determining equations for the nonclassical symmetries of higher order partial differential equations be derived by imposing a condition of pure compatibility? In the case of quasilinear evolutionary equations, Arrigo and Beckham [1] have shown that the nonclassical point symmetries can be recovered by seeking compatibility with first order quasilinear PDEs. As for compatibility with equations that are nonlinear and/or higher order, further study is required and is a topic of future work.

\section{Acknowledgements}

The author gratefully acknowledges the financial support of the University Research Council at the University of Central Arkansas.

\section{References}

[1] Arrigo D J and Beckham J R, Nonclassical symmetries of evolutionary partial differential equations and compatibility, J. Math. Anal. Appl. 289, 55-65 (2004).

[2] Bluman G W and Cole J D The general similarity solution of the heat equation, J. Math. Phys. 18, 1025-1042 (1969).

[3] Bluman G and Kumei S, Symmetries and Differential Equations, Springer- Verlag, 1989.

[4] Borovskikh A V, Group classification of the eikonal equation for a 3-dimensional inhomogeneous medium,Sbornik: Mathematics 195, 479-520 (2004).

[5] Herrera J J E, Minzoni A and Ondarza R, Reaction-diffusion equations in one-dimension: Particular solutions and relaxation, Physica D 57, 249-266 (1992).

[6] Hill J M, Avagliano A J and Edwards M P, Some exact results for nonlinear diffusion with absorption, IMA J. Appl. Math. 48, 283-304 (1992).

[7] Hydon P, Symmetry Methods for Differential Equations, Cambridge University Press, 2000.

[8] Keener J P, An eikonal-curvature equation for action potential propagation in myocardium, J. Math. Biol. (1991), 29, 629-651.

[9] Kells L M, Elementary Differential Equations, McGraw-Hill, New York, 1932.

[10] Kinsler L E, Frey A R, Cooper A B and Sanders J V, Fundementals of Acoustics, Wiley, 2000.

[11] Whitham G B, Linear and Nonlinear Waves, Wiley 1974.

[12] Wilhelmsson H, Solution of reaction-diffusion equations describing dynamic evolution towards explosive localized states, Phys. Rev. A 38, 2667-2670 (1988). 